\title{
Reflection coefficient null of acoustic waves at a liquid-anisotropic- solid interface
}

\author{
Orhan Arıkan \\ Electrical Engineering Department, University of Illinois at Urbana-Champaign, Urbana, Illinois 61801 \\ Emre Telatar \\ Electrical Engineering Department, Massachusetts Institute of Technology, Cambridge, Massachusetts 02139 \\ Abdullah Atalar \\ Electrical and Electronics Engineering Department, Bilkent University, Ankara, Turkey
}

(Received 2 February 1988; accepted for publication 22 August 1988)

\begin{abstract}
The reflection coefficient of acoustic waves incident on a liquid-solid interface from the liquid side is numerically calculated for a general anisotropic solid oriented in any arbitrary direction. The reflection coefficient depends, in general, on polar and azimuthal angles of incidence.

Results are presented for various crystalline materials of different symmetry classes. A null in the reflection coefficient amplitude is detected at a particular incidence angle for every anisotropic material that supports pseudosurface waves. The null in the reflection corresponds to the excitation of the pseudosurface wave on the interface surface, and it has typically a very narrow angular aperture. The angular position of the null is very critical and highly dependent on the elastic parameters of the anisotropic solid.
\end{abstract}

PACS numbers: 43.20.Fn, $43.35 . \mathrm{Cg}$

\section{INTRODUCTION}

Although the reflection-refraction problem at a liquidisotopic-solid interface has been intensively studied, ${ }^{1-3}$ the reflection-refraction problem at a liquid-anisotropic-solid interface remains relatively untouched because of the increasing complexity of analytic expressions and numerical difficulties. The theoretical aspects of the reflection-refraction problem between two anisotropic solids were reviewed by Henneke ${ }^{4}$ with a discussion on critical-angle phenomena. ${ }^{5}$ Some numerical calculations for special cases were given by Auld. ${ }^{6}$ Jones and Henneke ${ }^{7}$ gave a numerical solution for reflection of elastic waves from a stress-free boundary in an anisotropic half-space. Atalar ${ }^{8}$ presented the numerical solution of the reflection coefficient at a liquid-cubic-solid interface. Somekh et al. ${ }^{9}$ have studied the reflection coefficient of anisotropic materials at a liquid interface for the purpose of understanding acoustic images obtained by acoustic microscopes. They have numerically calculated the reflection coefficients for some materials and applied the results for the interpretation of contrast in acoustic images. Crean and Waintal ${ }^{10}$ have calculated the Rayleigh wave velocity on anisotropic substrates for the same purpose. Velasco and Garcia-Moliner ${ }^{11}$ have given a Green's function analysis to calculate surface waves in anisotropic cubic crystals. Rokhlin et al. ${ }^{12,13}$ have calculated the reflection and refraction of elastic waves at the planar interface of two generally anisotropic solids.

In this article, the results of a study on the reflection of plane acoustical waves at a planar liquid-anisotropic solid interface are presented. The solution is for the most general case, that is, the anisotropic solid can be of any symmetry class and the interface surface could be selected as any desired plane of the crystal.

\section{CALCULATION OF THE REFLECTION COEFFICIENT}

In this section, the acoustical wave equation and the plane-wave solutions in anisotropic media will be considered, the boundary conditions will be examined, and a solution method for the reflection-refraction problem at a liquid-anisotropic-solid interface will be derived. For simplicity, acoustical attenuation and piezoelectricity of both media are neglected. Both media are assumed to be of infinite extent and the interface is assumed to be an infinite plane. Due to the complexity of the expressions, an analytical solution is not possible, hence, a numerical approach is attempted. The analytic expressions will be abandoned only at the point where no more analytical manipulations are possible. The remaining part of the problem will be solved by numerical means. Although this derivation may be found elsewhere, ${ }^{4}$ we will give it for the sake of completeness and to bring clarity to notation.

Following the notation of Auld, ${ }^{6}$ consider the acoustic field equations and the constitutive relations in a nonpiezoelectric material:

$$
\mathbf{S}=\nabla_{s} \mathbf{u}, \quad \mathbf{T}=\mathbf{c}: \mathbf{S}, \quad \nabla \cdot \mathbf{T}=\rho \frac{\partial^{2} \mathbf{u}}{\partial t^{2}}-\mathbf{F},
$$

where $S, T$, and $c$ are the strain, stress, and stiffness constant matrices, respectively, in the abbreviated subscript notation. With this notation, $S$ and $T$ become 6 by 1 matrices and $c$ becomes a 6 by 6 matrix with, in general, 21 independent constants, $u$ and $\mathbf{F}$ vectors represent the particle displacement and the body force field in the material, $\rho$ is the density of the material, $\nabla_{s}$ is the symmetric gradient operator, and $\boldsymbol{\nabla}$. is the divergence operator. The latter are given by 


$$
\nabla_{s}=\left[\begin{array}{ccc}
\frac{\partial}{\partial x} & 0 & 0 \\
0 & \frac{\partial}{\partial y} & 0 \\
0 & 0 & \frac{\partial}{\partial z} \\
0 & \frac{\partial}{\partial z} & \frac{\partial}{\partial y} \\
\frac{\partial}{\partial z} & 0 & \frac{\partial}{\partial x} \\
\frac{\partial}{\partial y} & \frac{\partial}{\partial x} & 0
\end{array}\right]=(\nabla \cdot)^{T}
$$

Since for a freely vibrating medium the body force $\mathbf{F}$ is zero, the three equations of Eq. (1) may be combined to give

$$
\left(\nabla \cdot \mathbf{c}: \nabla_{s}\right) \mathbf{u}=\frac{\partial^{2} \mathbf{u}}{\partial t^{2}},
$$

which is a wave equation. The wave equation can be solved analytically only for a limited number of cases. The simplest case involves the plane-wave solution. If we introduce the phasor notation for plane waves, we can write the solution for the particle displacement field as

$$
\mathbf{u}=\operatorname{Re}\{A \mathbf{a} \exp [j(\omega t-\mathbf{k} \cdot \mathbf{r})]\},
$$

where $A$ is the amplitude of the plane wave, $\mathbf{a}$ is the unit polarization vector of the plane wave, $\omega$ is the angular frequency, $\mathbf{k}$ is the wave vector pointing in the propagation direction, and $\mathbf{r}$ is the position vector. With $\exp (j \omega t)$ time dependence, we may replace the operator $\partial / \partial x_{i}$ with $-j k_{i}$ and $d / d t$ by $j \omega$. Then, the symmetric gradient operator is reduced to

$\nabla_{s}=-j\left[\begin{array}{ccc}k_{x} & 0 & 0 \\ 0 & k_{y} & 0 \\ 0 & 0 & k_{z} \\ 0 & k_{z} & k_{y} \\ k_{z} & 0 & k_{x} \\ k_{y} & k_{x} & 0\end{array}\right]=-j k\left[\begin{array}{ccc}l_{x} & 0 & 0 \\ 0 & l_{y} & 0 \\ 0 & 0 & l_{z} \\ 0 & l_{z} & l_{y} \\ l_{z} & 0 & l_{x} \\ l_{y} & l_{x} & 0\end{array}\right]$,

where $l_{x}, l_{y}$, and $l_{z}$ are the direction cosines of $\mathbf{k}$. With the definition

$$
\boldsymbol{\nabla}_{s}=(\boldsymbol{\nabla} \cdot)^{T}=-j k \mathbf{L},
$$

Eq. (2) becomes

$$
-k^{2} \mathbf{L}^{T} \mathbf{c L a}=-\rho \omega^{2} \mathbf{a}
$$

or

$$
\left[k^{2} \mathbf{L}^{T} \mathbf{c L}-\rho \omega^{2} \mathbf{I}\right] \mathbf{a}=0,
$$

where $I$ is the third-order identity matrix. The matrix $\mathbf{L}^{T} \mathbf{c L}$ is called the Christoffel matrix and is denoted by $\Gamma\left(l_{x}, l_{y}, l_{z}\right)$. Note that the Christoffel matrix depends only on the stiffness constants and the propagation direction. Equation (3) is called the Christoffel equation. ${ }^{6}$ To obtain nontrivial solutions for $\mathbf{a}$ in Eq. (3), the determinant must be zero:

$$
\left|k^{2} \mathbf{\Gamma}-\rho \omega^{2} \mathbf{I}\right|=0 \text {. }
$$

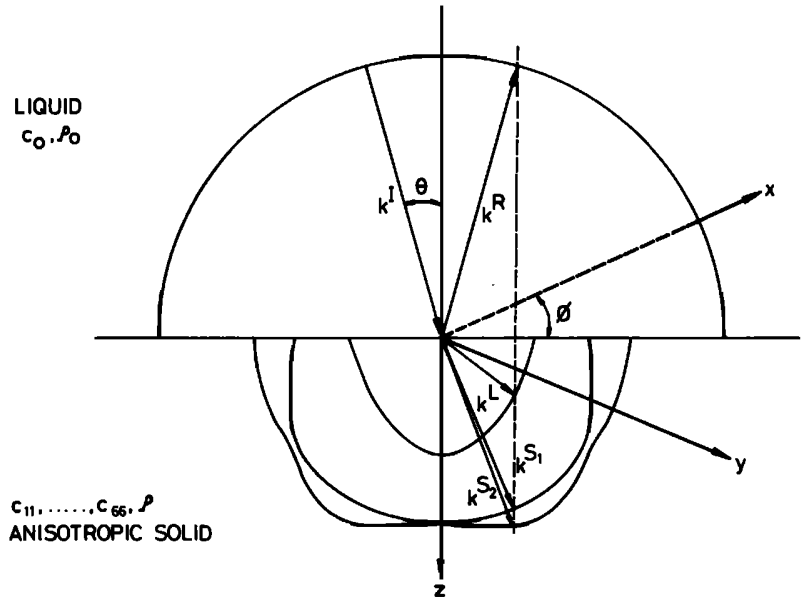

FIG. 1. Scattering geometry suitable for the liquid-anisotropic-solid reflection problem. Wave vectors along with slowness surfaces are shown. Interface is the $x-y$ plane.

Hence, we see that $\rho \omega^{2} / k^{2}$ are the eigenvalues of $\Gamma$ and $a$ is an eigenvector of $\Gamma$. We observe that, since $\Gamma$ is a 3 by 3 matrix, in general, there exist three different solutions for $\mathbf{k}$ and $\mathbf{a}$, corresponding to the same direction of propagation, which implies the existence of three different wave modes propagating in the same direction with different polarization vectors and velocities. Moreover, since $\Gamma$ is symmetric, the polarization vectors corresponding to distinct $k$ values are orthogonal. Also, Eq. (3) can be described entirely by the variable $\mathbf{k} / w$, which is called slowness. The slowness surfaces are defined to be the loci of the end points of the vector $\mathbf{k} / w$, and they prove to be useful in the solution of the reflectionrefraction problem. ${ }^{6}$

We now consider the reflection-refraction problem depicted in Fig. 1. The boundary between the liquid medium and the anisotropic solid is the $x-y$ plane. We assume a unit amplitude plane wave striking the interface with an angle $\theta$ and with a wave vector $\mathbf{k}^{\mathbf{l}}$. The angle between the plane of incidence and the $x$ axis is $\phi$. We write the particle displacement field of the incident wave in phasor form with $\exp (j \omega t)$ time dependence suppressed as

$$
\mathbf{u}^{\mathbf{I}}=\left(\mathbf{k}^{\mathbf{I}} / k_{0}\right) \exp \left(-j \mathbf{k}^{\mathbf{I}} \cdot \mathbf{r}\right),
$$

where $k_{0}$ is the wavenumber in the liquid. Since the incident medium is liquid, the only reflected mode is a longitudinal wave. On the other hand, in the anisotropic solid there can be three refracted modes: One quasilongitudinal (QL) and two quasishear (QS) waves. The corresponding particle displacement fields can be written as

$$
\begin{aligned}
& \mathbf{u}^{\mathbf{R}}=\boldsymbol{R}\left(\mathbf{k}^{\mathbf{R}} / k_{0}\right) \exp \left(-j \mathbf{k}^{\mathbf{R}} \cdot \mathbf{r}\right), \\
& \mathbf{u}^{\mathbf{L}}=L \mathbf{a}^{\mathbf{L}} \exp \left(-j \mathbf{k}^{\mathbf{L}} \cdot \mathbf{r}\right),
\end{aligned}
$$

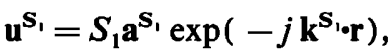

$$
\begin{aligned}
& \mathbf{u}^{\mathbf{S}_{2}}=S_{2} \mathbf{a}^{\mathbf{S}_{2}} \exp \left(-j \mathbf{k}^{\mathbf{S}_{2} \mathbf{r}}\right),
\end{aligned}
$$

where $\mathbf{k}^{\mathbf{R}}, \mathbf{k}^{\mathbf{L}}, \mathbf{k}^{\mathbf{S}_{1}}, \mathbf{k}^{\mathbf{S}_{2}}$ and $R, L, S_{1}, S_{2}$ are the wave vectors and the complex amplitudes of the reflected wave in the liquid and of the $\mathrm{QL}$ and two $\mathrm{QS}$ waves in the solid, respectively. Here, $\mathbf{a}^{\mathbf{L}}, \mathbf{a}^{\mathbf{s}_{1}}$, and $\mathbf{a}^{\mathbf{s}_{2}}$ stand for the unit polarization vectors of the refracted waves. 
The boundary conditions at the interface can be stated as follows. Since the liquid moves parallel to the interface plane freely, the condition for particle displacement continuity holds only for the normal component of $u$ :

$$
\mathbf{u} \cdot \hat{\mathbf{n}}=\mathbf{u}^{\prime} \cdot \hat{\mathbf{n}} \text {, }
$$

where $\mathbf{u}$ and $\mathbf{u}^{\prime}$ are the particle displacements in the liquid and in the solid, respectively, and $\hat{n}$ is the unit normal vector of the interface. This boundary condition gives

$$
\left.\left(u_{z}^{I}+u_{z}^{R}\right)\right|_{z=0}=\left.\left(u_{z}^{L}+u_{z}^{S_{1}}+u_{z}^{S_{2}}\right)\right|_{z=0},
$$

where $u_{z}$ is the $z$ component of the particle displacement and the superscripts denote the type of the wave as before. The second set of boundary conditions arise from dynamical constraints:

$$
\mathbf{T} \cdot \hat{\mathbf{n}}=\mathbf{T}^{\prime} \cdot \hat{\mathbf{n}},
$$

where $\mathbf{T}$ and $\mathbf{T}^{\prime}$ are the respective stress tensors of the two media. From this boundary condition, we find

$$
\left.\left(T_{J}^{I}+T_{J}^{R}\right)\right|_{z=0}=\left.\left(T_{J}^{L}+T_{J}^{S_{1}}+T_{J}^{S_{2}}\right)\right|_{z=0}, \quad J=3,4,5,
$$

where the stress components are shown in abbreviated subscript notation.

An implication of Eq. (4) is the conservation of the phase velocity parallel to the boundary:

$$
\mathbf{k} \times \hat{\mathbf{n}}=\mathbf{k}^{\prime} \times \hat{\mathbf{n}},
$$

or

$$
\begin{aligned}
& k_{x}^{I}=k_{x}^{R}=k_{x}^{L}=k_{x}^{S_{1}}=k_{x}^{S_{2}}=l_{x} k_{0}, \\
& k_{y}^{I}=k_{y}^{R}=k_{y}^{L}=k_{y}^{S_{1}}=k_{y}^{S_{2}}=l_{y} k_{0},
\end{aligned}
$$

which is also known as Snell's law. Although Snell's law determines readily the $x$ and $y$ components for the wave vectors of reflected $\left(k^{R}\right)$ and refracted longitudinal $\left(k^{\mathbf{L}}\right)$, and shear $\left(\mathbf{k}^{\mathbf{S}_{1}}, \mathbf{k}^{\mathbf{S}_{2}}\right)$ modes, the $z$ components of wave vectors and, hence, the propagation directions are more difficult to ascertain.

For this purpose, the Christoffel equation should be modified for the solution of $k_{z}$. The Christoffel equation may be rewritten as:

$$
\begin{gathered}
{\left[\begin{array}{cccccc}
k_{x} & 0 & 0 & 0 & k_{z} & k_{y} \\
0 & k_{y} & 0 & k_{z} & 0 & k_{x} \\
0 & 0 & k_{z} & k_{y} & k_{x} & 0
\end{array}\right]\left[c_{i j}\right]} \\
\times\left[\begin{array}{ccc}
k_{x} & 0 & 0 \\
0 & k_{y} & 0 \\
0 & 0 & k_{z} \\
0 & k_{z} & k_{y} \\
k_{z} & 0 & k_{x} \\
k_{y} & k_{x} & 0
\end{array}\right]-\rho \omega^{2} \mathbf{I} \mid=0,
\end{gathered}
$$

which leads in general to a sixth-order equation in $k_{z}$. Equation (6) can be written explicitly as follows:

$$
\begin{aligned}
& -\rho^{3} \omega^{6}+\rho^{2} \omega^{4}\left(h_{1}+h_{2}+h_{3}\right) \\
& \quad-\rho \omega^{2}\left(h_{1} h_{2}+h_{2} h_{3}+h_{3} h_{1}-h_{4}^{2}-h_{5}^{2}-h_{6}^{2}\right) \\
& \quad+h_{1} h_{2} h_{3}+2 h_{4} h_{5} h_{6}-h_{1} h_{4}^{2}-h_{2} h_{5}^{2}-h_{3} h_{6}^{2}=0,
\end{aligned}
$$

where

$$
h_{i}=a_{i} k_{z}^{2}+b_{i} k_{z}+c_{i}, \quad i=1, \ldots, 6,
$$

and

$$
\begin{array}{ll}
a_{1}=c_{55}, & b_{1}=2\left(c_{15} k_{x}+c_{56} k_{y}\right), \\
a_{2}=c_{44}, & b_{2}=2\left(c_{46} k_{x}+c_{24} k_{y}\right), \\
a_{3}=c_{33}, & b_{3}=2\left(c_{35} k_{x}+c_{34} k_{y}\right), \\
a_{4}=c_{34}, & b_{4}=\left(c_{36}+c_{45}\right) k_{x}+\left(c_{23}+c_{44}\right) k_{y}, \\
a_{5}=c_{35}, & b_{5}=\left(c_{55}+c_{13}\right) k_{x}+\left(c_{45}+c_{36}\right) k_{y}, \\
a_{6}=c_{45}, & b_{6}=\left(c_{14}+c_{56}\right) k_{x}+\left(c_{46}+c_{25}\right) k_{y}, \\
c_{1}=c_{11} k_{x}^{2}+c_{66} k_{y}^{2}+2 c_{16} k_{x} k_{y}, \\
c_{2}=c_{66} k_{x}^{2}+c_{22} k_{y}^{2}+2 c_{26} k_{x} k_{y}, \\
c_{3}=c_{55} k_{x}^{2}+c_{44} k_{y}^{2}+2 c_{45} k_{x} k_{y}, \\
c_{4}=c_{56} k_{x}^{2}+c_{24} k_{y}^{2}+\left(c_{25}+c_{46}\right) k_{x} k_{y}, \\
c_{5}=c_{15} k_{x}^{2}+c_{46} k_{y}^{2}+\left(c_{14}+c_{56}\right) k_{x} k_{y}, \\
c_{6}=c_{16} k_{x}^{2}+c_{26} k_{y}^{2}+\left(c_{66}+c_{12}\right) k_{x} k_{y} .
\end{array}
$$

The above-mentioned boundary conditions are not sufficient for the correct solution of the problem. Another set of conditions is necessary to make sure that the nonevanescent refracted modes carry energy into the solid medium and the evanescent modes decay into the solid medium as a function of depth. The $\mathbf{k}$ vector direction is not a correct measure of the energy propagation direction, instead the direction of Poynting vector $\mathbf{P}$ should be considered. ${ }^{4}$ For a refracted mode solution to be a valid one, its Poynting vector must be directed into the solid. This vector does not generally coincide with the normal to the wave front in anisotropic media, but it is in the direction of the gradient of the slowness surface. In our case, with anisotropic media at the positive $z$ region, the $z$ component of the Poynting vector is the only component that is important. In phasor notation, this component is given by

$$
P_{z}=-\frac{1}{2}\left(v_{x}^{*} T_{5}+v_{y}^{*} T_{4}+v_{z}^{*} T_{3}\right),
$$

where $v_{i}^{*}$ 's are the conjugates of the respective particle velocities. For nonevanescent modes, $\boldsymbol{P}_{z}$ must have a positive real part in order to have a power flow with a positive component in the $z$ direction. For evanescent modes, it has to have a negative imaginary part to get a wave that cannot carry any energy and that vanishes as $z$ approaches infinity. With these constraints, the six solutions found for $k_{z}$ from the previous part are reduced to three. Note that there are exactly three $k_{z}$ 's satisfying the $P_{z}$ conditions.

The solution algorithm may be given as follows: Given the stiffness constants of the solid and the angle of incidence, the sixth-order equation in terms of $k_{z}$ is constructed using Eq. (7). This equation can be solved by numerical means for $k_{z}$, resulting in the six $k_{z}$ values that are not necessarily distinct. Then Eq. ( 3 ) is contructed and the normalized polarization vectors $\left(\mathbf{a}^{\mathbf{L}}, \mathbf{a}^{\mathbf{s}_{1}}, \mathbf{a}^{\mathbf{s}_{2}}\right)$, are found. For each of these $k_{z}$ 's and the corresponding polarization vectors, the $z$ components of the Poynting vector, is calculated by Eq. (8). The roots that have $\mathbf{P}$ vectors pointing to the liquid media or those leading to exponentially growing waves should be omitted. 
Knowing the wave vectors and unit polarizations of all reflected and refracted modes, the four boundary conditions may be written in terms of the unknown amplitudes, $R, L$, $S_{1}$, and $S_{2}$. From these four linear equations [Eqs. (4) and (5) ], the unknown amplitudes of the reflected and refracted modes can be solved.

There may be numerical difficulties for angles near the normal incidence and near the critical angles where the problem becomes ill-conditioned. Special precautions must be taken for such cases to obtain the correct numerical results. These include the ordering of roots, and reducing the degree of the equations to be solved if roots are very close to each other.

\section{RESULTS}

A FORTRAN program was developed to carry out the numerical calculations necessary for the evaluation of the reflection coefficient $R$. The program can handle materials with arbitrary stiffness matrices of 21 constants, so materials with arbitrary orientation can be handled with proper trans-
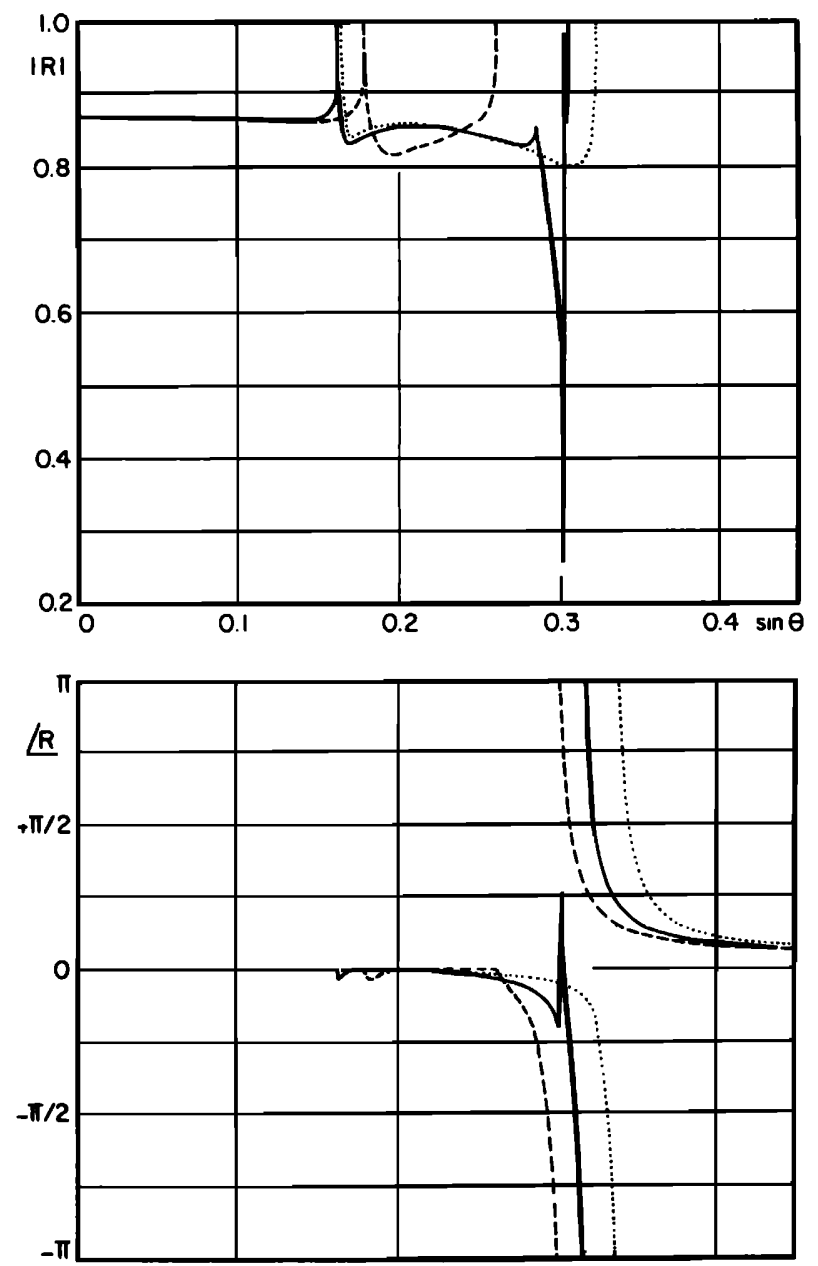

FIG. 2. Reflection coefficient amplitude and phase at a $\mathrm{H}_{2} \mathrm{O}-\mathrm{Si}(011)$ interface as a function of incidence angle for azimuthal angles of $0^{\circ}$ (dotted), $44.81^{\circ}$ (solid), and $90^{\circ}$ (dashed). The constants for $\mathrm{H}_{2} \mathrm{O}$ are $c_{11}=2.277 \mathrm{E} 9$ and $\rho=1000$.
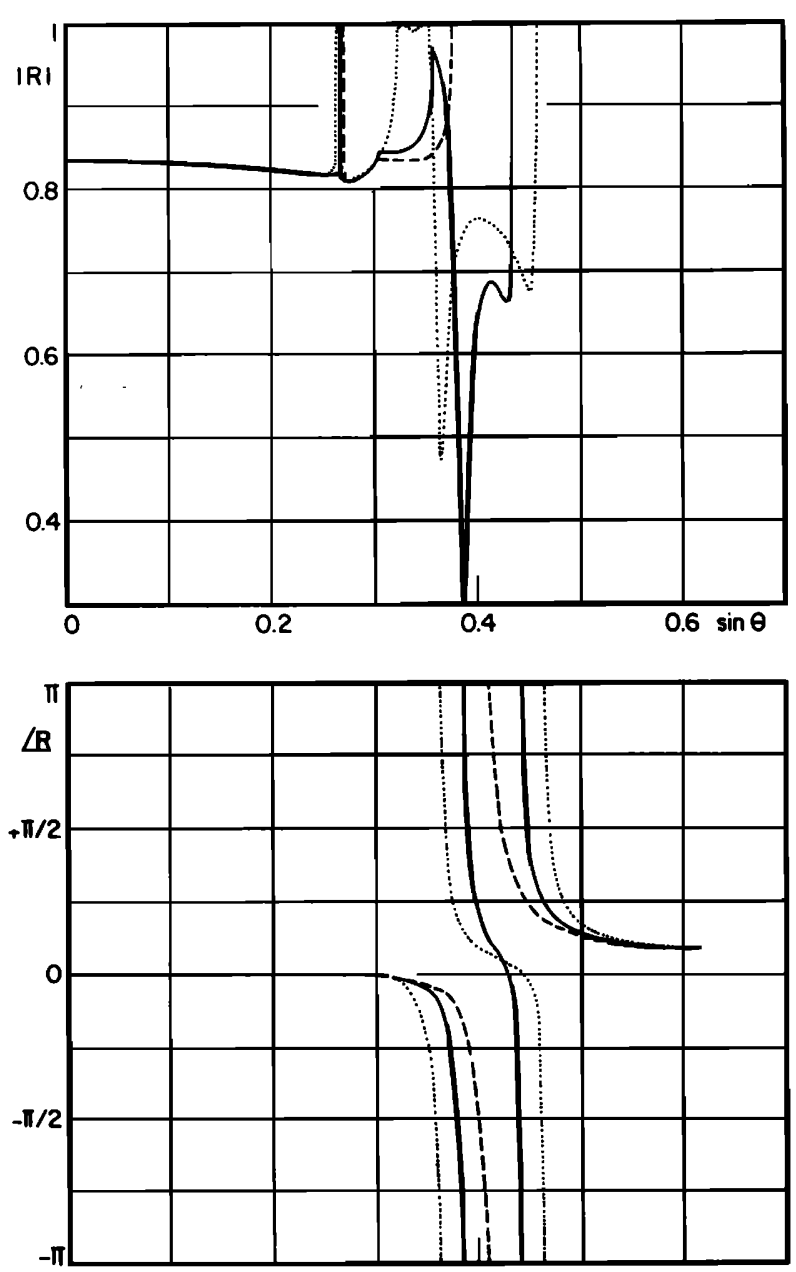

FIG. 3. Reflection coefficient amplitude and phase at a $\mathrm{H}_{2} \mathrm{O}-$ quartz $(001)$ interface as a function of incidence angle for azimuthal angles of $0^{\circ}$ (dotted), $17.91^{\circ}$ (solid), and $30^{\circ}$ (dashed).

formation of the stiffness matrix by multiplication with the Bond matrices. ${ }^{6}$ The program was tested against the materials whose reflection coefficients were previously calculated. ${ }^{8,9}$ The results were consistent with those previous calculations within the expected precision which was about $0.1 \%$. Obviously, these tests do not prove that the program works correctly for all cases, nevertheless running a number of different cases assured us that the program is reliable.

In Fig. 2, the reflection coefficient $R$, amplitude, and phase at the $\mathrm{H}_{2} \mathrm{O}-\mathrm{Si}$ interface for the (011) plane of $\mathrm{Si}$ are shown as a function of $\sin \theta$ for $\phi=0^{\circ}, 44.81^{\circ}$, and $90^{\circ}$. Here, $\theta$ and $\phi$ are the sperical coordinates (polar and azimuthal angles, respectively) where $\phi$ is the angle between the incidence plane and the [100] axis. Concentrating on the $\phi=44.81^{\circ}$ curve in the figure, we can identify the following regions: for $\sin \theta<0.1622$ all three wave modes, namely one QL and two QS waves, propagate into the solid, and the reflection coefficient is approximately constant. Above $\sin \theta=0.1622$ the QL wave can no longer be excited, and until $\sin \theta=0.2857$ only two QS waves are present. While $\sin \theta$ is between 0.2857 and 0.3059 , only one QS wave is excited. At $\sin \theta=0.3016$ there is a minimum in the reflec- 
tion coefficient. Note that, for such an incidence angle, more than $94 \%$ of the incident energy is transmitted into the solid. We also observe that, for $\sin \theta=0.3159$, there is a transition in the phase of the reflection coefficient corresponding to a Rayleigh wave excitation.

In Fig. 3, the reflection coefficient versus $\sin \theta$ plots for the $\mathrm{H}_{2} \mathrm{O}$-quartz (001) interface for $\phi=0^{\circ}, 17.91^{\circ}$, and $30^{\circ}$ are shown. Focusing on the $\phi=17.91^{\circ}$ curve, we observe a phase transition at $\sin \theta=0.3848$. The reflection coefficient amplitude has a minimum value of 0.2950 at $\sin \theta=0.3857$. At $\sin \theta=0.4408$, there is another phase transition and above this angle no wave propagates into the solid. Note that two phase transitions occur and the amplitude minimum is nearly at the first transition.

Figure 4 presents the reflection coefficient versus $\sin \theta$ plots for the $\mathrm{H}_{2} \mathrm{O}$-quartz interface for $\phi=0^{\circ}, 45^{\circ}$, and $90^{\circ}$ at the (100) plane. At this plane of quartz, a minimum is observed for the $\phi=45^{\circ}$ curve.

In Figs. 2-4, for particular values of $\phi$, we observe a dip in the amplitude of the reflection coefficient at a polar angle corresponding to a single QS wave excitation in the solid medium. At this angle there is an almost complete power


FIG. 4. Reflection coefficient magnitude at a $\mathrm{H}_{2} \mathrm{O}$-quartz (100) interface as a function of incidence angle for azimuthal angles of $0^{\circ}$ (dotted), $45^{\circ}$ (solid), and $90^{\circ}$ (dashed).

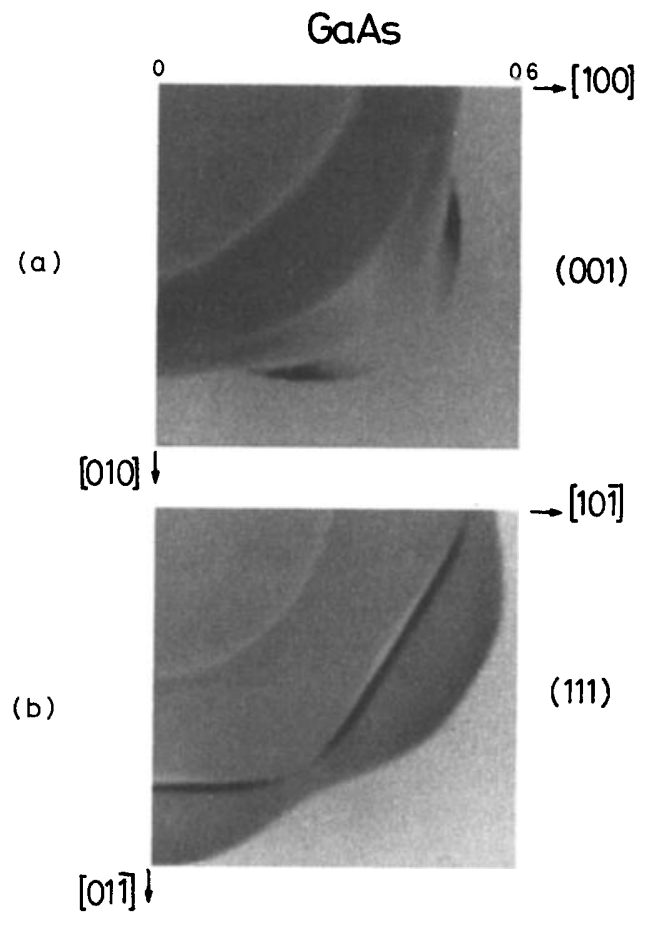

FIG. 5. Reflection coefficient magnitude at a $\mathrm{H}_{2} \mathrm{O}-\mathrm{GaAs}$ interface as a function of direction cosines (horizontal and vertical axes $0<\sin \theta<0.6$ ) plotted in two dimensions, (a) (100) plane, (b) (111) plane.

transfer from the low-impedance liquid medium to the highimpedance solid medium.

Complete presentation of results is rather difficult in the way shown in Figs. 2-4. A more compact presentation is possible if three-dimensional views are used. At the third

(a)

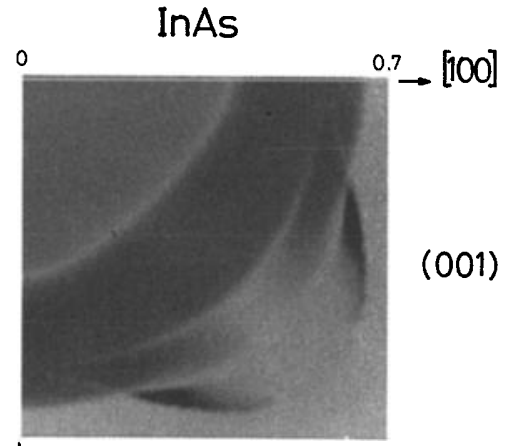

(b)

$[010]$



FIG. 6. Reflection coefficient magnitude at a $\mathrm{H}_{2} \mathrm{O}-$ InAs interface as a function of direction cosines (horizontal and vertical axes $0<\sin \theta<0.7$ ) plotted in two dimensions, (a) (100) plane, (b) (111) plane. 

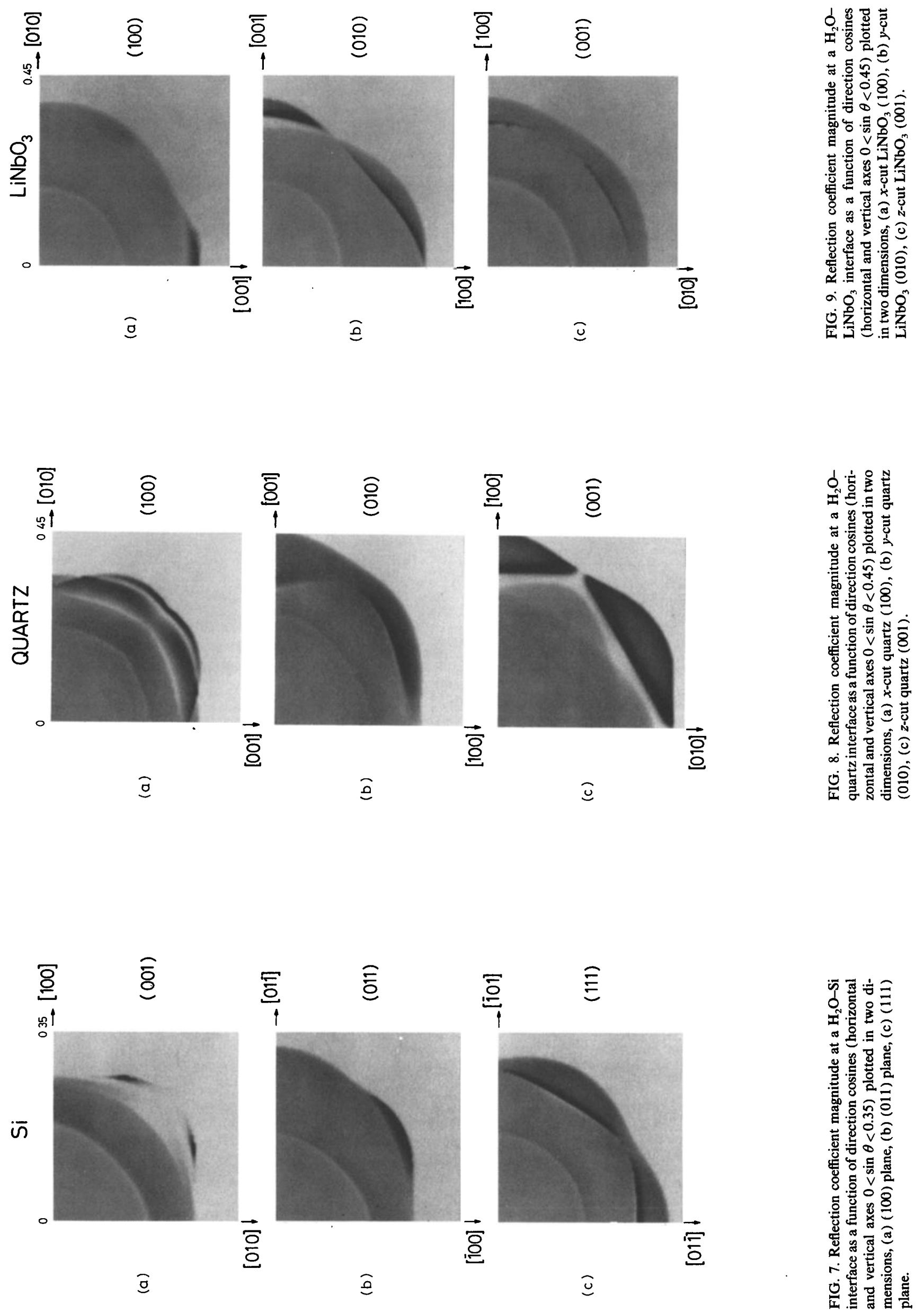


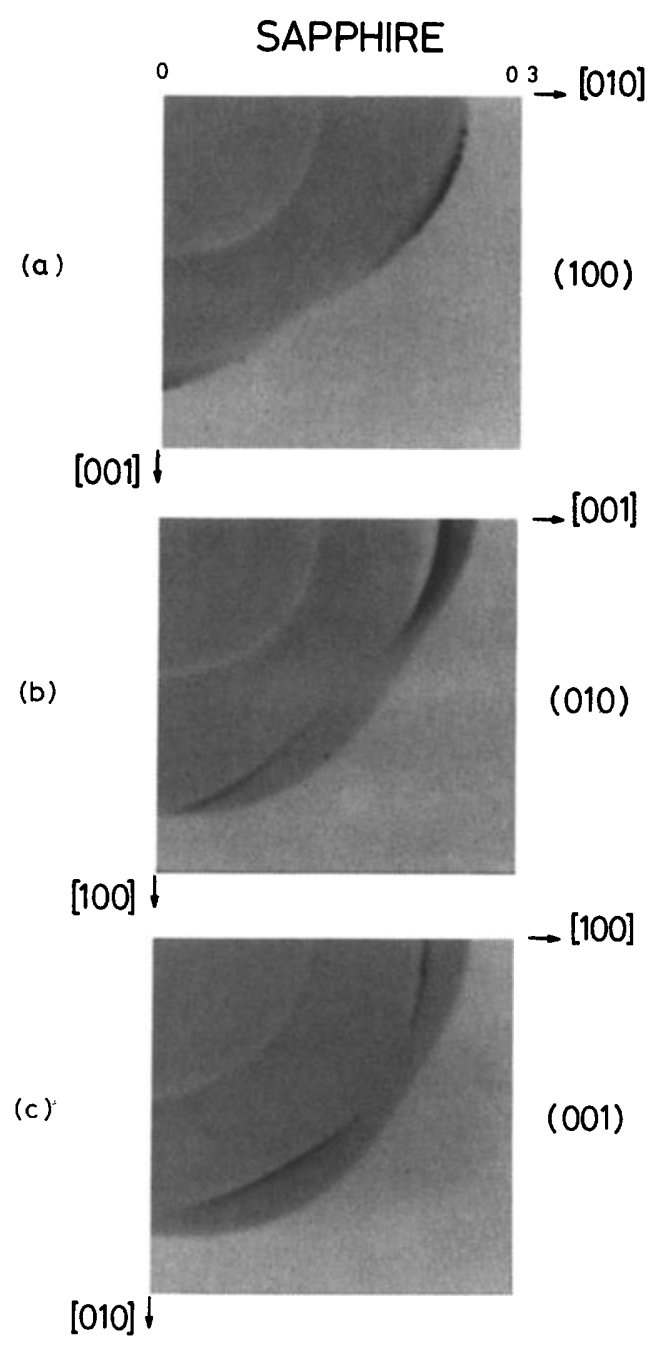

FIG. 10. Reflection coefficient magnitude at a $\mathrm{H}_{2} \mathrm{O}$-sapphire interface as a function of direction cosines (horizontal and vertical axes $0<\sin \theta<0.30$ ) plotted in two dimensions, (a) (100) plane, (b) (010) plane, (c) (001) plane.

dimension, it is possible to use gray shadings as obtained on a grey scale graphics monitor. Figure 5 is such a presentation for the reflection coefficient amplitude at the interface between $\mathrm{H}_{2} \mathrm{O}$ with (001) and (111) planes of $\mathrm{GaAs}$ crystal.
TABLE II. Minimum reflection angles for some hexagonal crystals at the $\mathrm{H}_{2} \mathrm{O}$ interface. Piezoelectricity neglected.

\begin{tabular}{lccc}
\hline \hline & \multicolumn{3}{c}{ Hexagonal materials } \\
Material & $\begin{array}{r}\text { (001) plane, } \mathrm{m}=[001], \begin{array}{c}\mathrm{n}=[100] \\
\sin \theta\end{array} \\
\phi\end{array}$ & $|R|$ \\
\hline $\mathrm{Ti}$ & 0.2812 & 12.83 & 0.846 \\
$\mathrm{ZnO}$ & 0.5252 & 17.74 & 0.854 \\
$\mathrm{BeO}$ & 0.2005 & 14.28 & 0.908 \\
$\mathrm{CdS}$ & 0.8284 & 24.94 & 0.632 \\
\hline
\end{tabular}

Calculations are performed on a 128 by 128 grid. In the figure the bright points correspond to a reflection coefficient that is close to unity whereas the dark points indicate a reflection coefficient close to zero. The critical angles are clearly seen as boundaries of different regions. Figures 6-10 are similar figures, which require extensive computation time, for the $\mathrm{H}_{2} \mathrm{O}$-InAs, $\mathrm{H}_{2} \mathrm{O}$-silicon, $\mathrm{H}_{2} \mathrm{O}$-quartz, $\mathrm{H}_{2} \mathrm{O}$ $\mathrm{LiNbO}_{3}$, and $\mathrm{H}_{2} \mathrm{O}$-sapphire interfaces at different crystallographic orientations of the solids. In all cases, the dark regions occur in a narrow path of direction cosines.

To find the position of the nulls - if they exist-we developed a minimization program using the steepest descent technique. Since the reflection coefficient has a highly complicated structure, it is possible to miss the real minimum and instead find a local minimum. We tried to find the null in the reflection coefficient magnitude for a number of materials. The constants of all the materials were taken from Auld. ${ }^{6}$ We summarize the results in Tables I-IV. They show the positions of nulls or minima for various materials at some crystallographic orientations. For cubic materials the anisotropy factor $\eta=2 c_{44} /\left(c_{11}-c_{12}\right)$ is also listed. In the tables, $\theta$ is the polar angle, which is between the incidence direction and the $m$ direction, and $\phi$ is the azimuth angle, which is between the incidence plane and the $\mathbf{n}$ direction, where $\mathbf{m}$ and $\mathbf{n}$ are as defined in the tables.

To show the angular extent of the nulls, we plotted the reflection coefficient at and around the nulls. Figures 11 and 12 show the reflection coefficient magnitude and phase for two cubic materials at their (111) planes. Typically, as the anisotropy factor $\eta$ increases, the angular extent of the null gets wider for (001) and (111) planes of cubic crystals. The

TABLE I. Minimum reflection angles for some cubic materials at the $\mathrm{H}_{2} \mathrm{O}$ interface. Piezoelectricity neglected.

\begin{tabular}{|c|c|c|c|c|c|c|c|c|c|c|}
\hline \multicolumn{2}{|c|}{$\begin{array}{c}\text { Cubic } \\
\text { materials }\end{array}$} & \multicolumn{3}{|c|}{$\begin{array}{c}(001) \text { plane } \\
\mathbf{m}=[001], \mathbf{n}=[100]\end{array}$} & \multicolumn{3}{|c|}{$\begin{array}{c}\text { (011) plane } \\
\mathbf{m}=[011], \mathbf{n}=[01 \overline{1}]\end{array}$} & \multicolumn{3}{|c|}{$\begin{aligned} & \text { (111) plane } \\
\mathbf{m}= & {[111], \mathbf{n}=[10 \overline{1}] }\end{aligned}$} \\
\hline Material & $\eta$ & $\sin \theta$ & $\phi$ & $|R|$ & $\sin \theta$ & $\phi$ & $|R|$ & $\sin \theta$ & $\phi$ & $|R|$ \\
\hline $\mathrm{Cu}$ & 3.18 & 0.6655 & 25.16 & 0.037 & 0.6800 & 40.84 & 0.704 & 0.5495 & 30.00 & 0.051 \\
\hline $\mathrm{Ni}$ & 2.63 & 0.5123 & $25: 66$ & 0.049 & 0.5317 & 40.72 & 0.765 & 0.4380 & 24.89 & 0.036 \\
\hline $\mathrm{Fe}$ & 2.41 & 0.4802 & 25.30 & 0.023 & 0.5020 & 40.57 & 0.739 & 0.4170 & 22.96 & 0.056 \\
\hline InAs & 2.08 & 0.6796 & 24.01 & 0.016 & 0.7111 & 42.45 & 0.402 & 0.6187 & 15.69 & 0.047 \\
\hline GaAs & 1.82 & 0.5301 & 24.93 & 0.001 & 0.5465 & 46.96 & 0.470 & 0.4742 & 20.37 & 0.014 \\
\hline $\mathbf{S i}$ & 1.56 & 0.2980 & 26.99 & 0.026 & 0.3019 & 44.88 & 0.179 & 0.2655 & 28.25 & 0.080 \\
\hline Al & 1.22 & 0.5030 & 32.37 & 0.090 & 0.4838 & 7.95 & 0.744 & 0.4700 & 29.99 & 0.290 \\
\hline $\mathrm{PbS}$ & 0.508 & 0.8100 & 0.00 & 0.710 & 0.7418 & 18.31 & 0.005 & 0.7301 & 9.263 & 0.302 \\
\hline
\end{tabular}


TABLE III. Minimum reflection angles for some tetragonal crystals at the $\mathrm{H}_{2} \mathrm{O}$ interface.

\begin{tabular}{ccccc}
\hline \hline & \multicolumn{4}{c}{ Tetragonal materials } \\
Material & \multicolumn{1}{c}{ (001) plane, } & $\mathbf{m}=[001]$, & $\mathbf{n}=[100]$ & \\
& $\beta$ & $\sin \theta$ & $\phi$ & $|R|$ \\
\hline $\mathrm{TeO}_{2}$ & 29.29 & 0.7433 & 28.65 & 0.009 \\
$\mathrm{TiO}_{2}$ & 4.06 & 0.2982 & 33.42 & 0.053 \\
\hline \hline
\end{tabular}

converse is true for (011) planes. Figure 13 depicts the reflection coefficient for a cubic material at its (011) plane. Figure 14 is a similar presentation for a tetragonal material at its $X-Y(001)$ plane. Notice that $\mathrm{TeO}_{2}$ is an unusually high anisotropic material, and the extent of the null is remarkably wide. Figures 15 and 16 show the reflection coefficient null for two common trigonal materials, quartz and sapphire. An important observation in all cases is that there is a phase transition of the reflection coefficient at the angle of minimum amplitude. This transition is different from the phase transition associated with the Rayleigh wave excitation that occurs at greater angles and at which all waves in the solid are evanescent. Both transitions are visible in Figs. 12 and 16. The phase transition at the amplitude minimum corresponds to a surface wave excitation as discussed in the next section.

\section{DISCUSSION}

The presence of the reflection coefficient nulls was shown to exist for (001) planes of some cubic crystals earlier. ${ }^{8}$ We now see that such angles exist for other planes of solids and are not limited to (001) faces of cubic crystals. The discussion given in Ref. 8 claimed that the null in the reflection coefficient would occur at an angle corresponding to an excitation of a pseudosurface wave. The pseudosurface waves were first encountered in surface-acoustic-wave $e^{14-15}$ and reflectivity experiments. ${ }^{16-18}$ The most complete treatment of the pseudosurface wave was given by $\mathrm{Lim}^{19}$ and Farnell. ${ }^{20}$ In contrast to the Rayleigh waves, which are surface waves, the pseudosurface waves are actually bulk waves that are traveling very close to the surface and, hence, acting like a surface wave radiating the energy of the wave into the bulk. They were also the subject of some recent investigations in conjunction with phonon imaging. ${ }^{21}$

The null angles in the reflection coefficient were first

TABLE IV. Minimum reflection angles for some trigonal crystals at the $\mathrm{H}_{2} \mathrm{O}$ interface. Piezoelectricity neglected.

\begin{tabular}{|c|c|c|c|c|c|}
\hline \multicolumn{6}{|c|}{ Trigonal materials } \\
\hline \multicolumn{2}{|c|}{ Material } & & $\sin \theta$ & $\phi$ & $|\boldsymbol{R}|$ \\
\hline Quartz (001), & $\mathbf{m}=[001]$ & $n=[100]$ & 0.3863 & 11.81 & 0.295 \\
\hline Quartz (100), & $\mathbf{m}=[100]$, & $\mathbf{n}=[010]$ & 0.3929 & 44.50 & 0.027 \\
\hline Quartz (010), & $\mathbf{m}=[010]$, & $\mathbf{n}=[001]$ & 0.3728 & 42.53 & 0.370 \\
\hline Sapphire (001), & $\mathbf{m}=[001]$ & $n=[100]$ & 0.2277 & 55.26 & 0.686 \\
\hline Sapphire (100), & $\mathbf{m}=[100]$, & $\mathbf{n}=[010]$ & 0.2340 & 42.49 & 0.619 \\
\hline Sapphire (010), & $\mathbf{m}=[010]$, & $\mathbf{n}=[001]$ & 0.2386 & -0.50 & 0.066 \\
\hline $\mathrm{LiNbO}_{3}(010)$ & $\mathbf{m}=[010]$, & $\mathbf{n}=[001]$ & 0.3988 & 166.86 & 0.044 \\
\hline
\end{tabular}

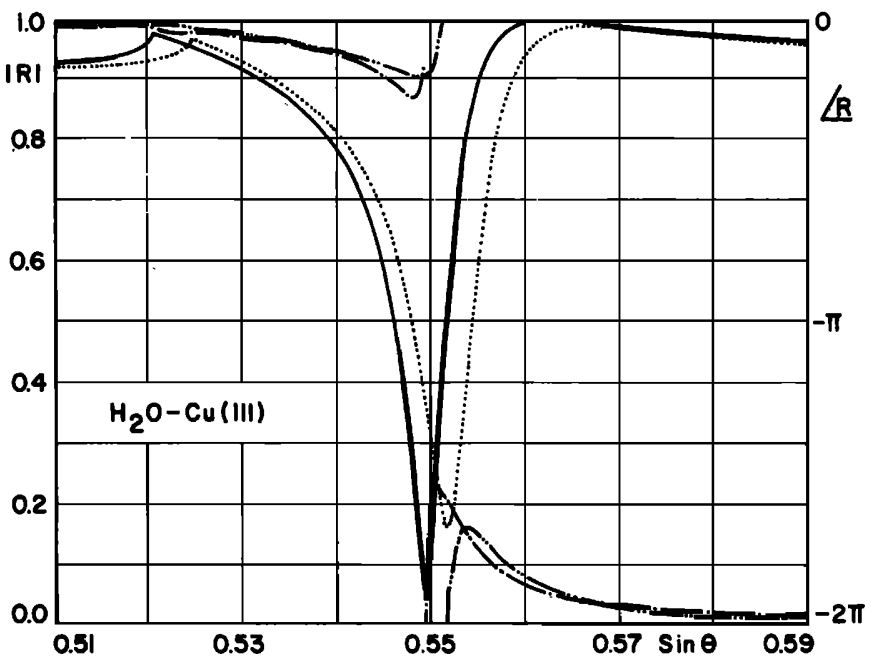

FIG. 11. Reflection coefficient magnitude at a $\mathrm{H}_{2} \mathrm{O}-\mathrm{Cu}$ (111) interface near the null as a function of incidence angle for azimuthal angles of $30^{\circ}$ (solid), $27^{\circ}$ (dotted), $33^{\circ}$ (dotted) and phase for $30^{\circ}$ (dash-dot), $27^{\circ}$ (dash-dot-dot), $33^{\circ}$ (dash-dot-dot).

observed experimentally by Rollins. ${ }^{16} \mathrm{He}$ noted that, for some orientations, the dip associated with the pseudosurface wave was much stronger than that related to the Rayleigh wave. The latter dip is strongly dependent on the absorption of Rayleigh waves and its physical interpretation is well understood. ${ }^{2}$ The first dip is due to the fact that incident longitudinal waves in the liquid medium is coupled almost completely into the pseudosurface waves. He reported the observation of complete nulls for the $Y-Z$ plane of quartz corresponding to the pseudosurface wave excitation.

Farnel1 ${ }^{20}$ indicated that the pseudosurface waves will exist on the (001) and (111) planes of the cubic crystals as long as the condition $\left(c_{11}-c_{12}\right) / 2<c_{44}$ is satisfied. Similar-

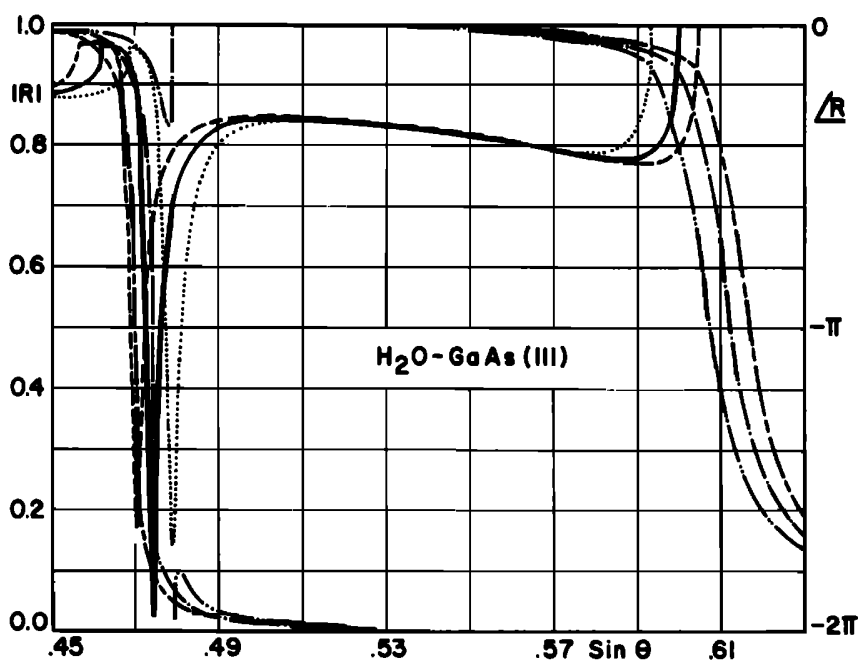

FIG. 12. Reflection coefficient magnitude at a $\mathrm{H}_{2} \mathrm{O}-\mathrm{GaAs}$ (111) interface near the null as a function of incidence angle for azimuthal angles of $17.37^{\circ}$ (dotted), $20.37^{\circ}$ (solid), $23.37^{\circ}$ (dashed) and phase for $17.37^{\circ}$ (dash-dotdot), $20.37^{\circ}$ (dash-dot), $23.37^{\circ}$ (dash-long dash). 


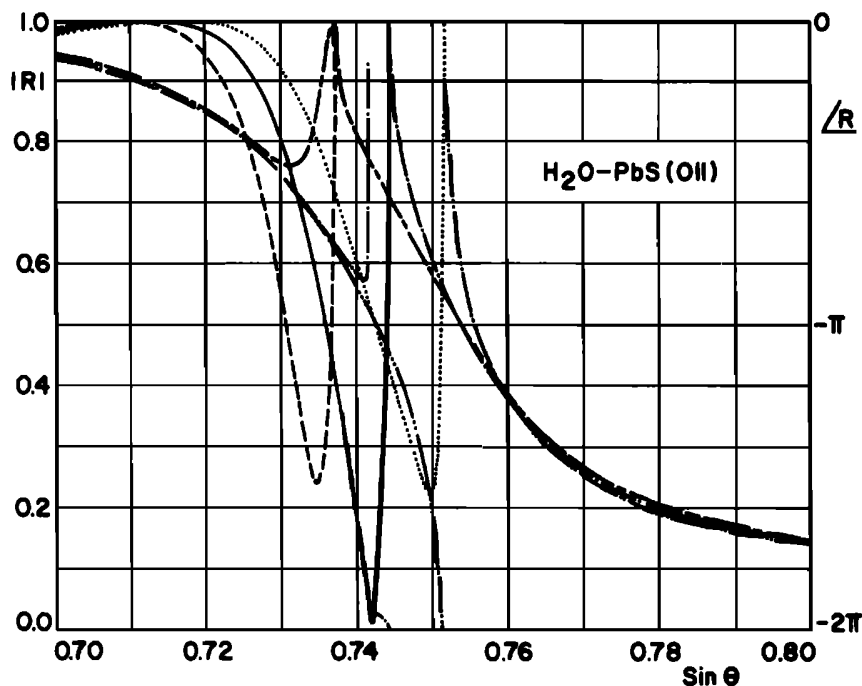

FIG. 13. Reflection coefficient magnitude at a $\mathrm{H}_{2} \mathrm{O}-\mathrm{PbS}(011)$ interface near the null as a function of incidence angle for azimuthal angles of $17.31^{\circ}$ (dotted), $18.31^{\circ}$ (solid), $19.31^{\circ}$ (dashed) and phase for $17.31^{\circ}$ (dash-dotdot), $18.31^{\circ}$ (dash-dot), $19.31^{\circ}$ (dash-long dash).

ly, the (011) planes of cubic crystals will support the pseudosurface waves, if $\left(c_{11}-c_{12}\right) / 2>c_{44}$ is satisfied. Inspection of Table I indicates that these conditions are indeed consistent with our findings. We find nulls in the $(001)$ and (111) planes of those cubic materials that have $\eta>1$, and in the (011) planes for which $\eta<1$. If the cubic materials is nearly isotropic (like $\mathrm{Al}$ ) there is a deep minimum, but it is not a complete null. Specifically, the (001) and (111) planes of crystalline copper support the pseudosurface waves and they were experimentally observed. ${ }^{17}$ The calculated position of nulls correspond to surface wave velocities of 2267 $\mathrm{m} / \mathrm{s}$ for the (001) plane and $2746 \mathrm{~m} / \mathrm{s}$ for the (111) plane.

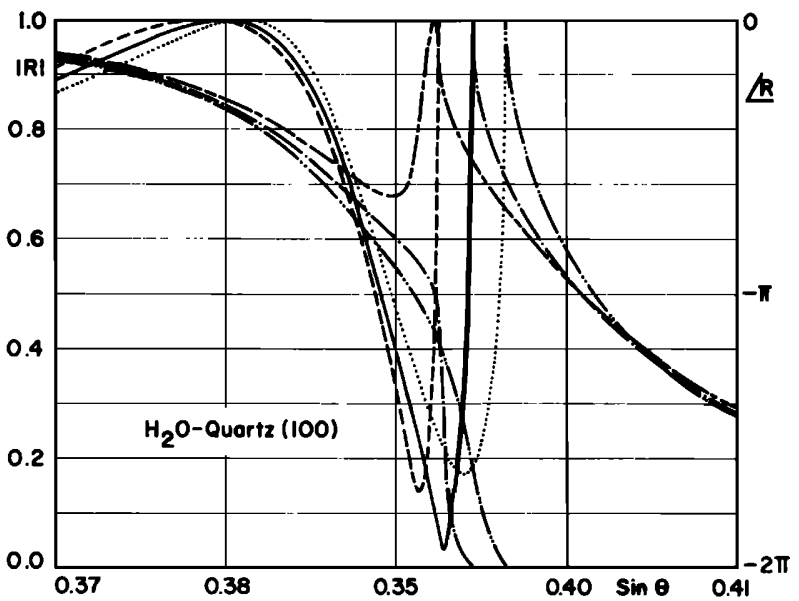

FIG. 15. Reflection coefficient magnitude at a $\mathrm{H}_{2} \mathrm{O}$-quartz (100) interface near the null as a function of incidence angle for azimuthal angles of $43.5^{\circ}$ (dotted), $44.5^{\circ}$ (solid), $45.5^{\circ}$ (dashed) and phase for $43.5^{\circ}$ (dash-dot-dot), $44.5^{\circ}$ (dash-dot), $45.5^{\circ}$ (dash-long dash).

These numbers and the azimuthal angles indeed agree very well with the measured and theoretically calculated velocities of the pseudosurface waves as shown in Figs. 1 and 2 of Ref. 17. The correspondence between the null angles and the pseudosurface waves can be more easily seen in Figs. 5-10. There the path followed by the dark regions maps the angular position ( $\sin \theta$ and $\phi$ ) of the pseudosurface waves. Existence of phase transitions at the amplitude minima as seen in Figs. 11-16 supports further the claimed association between the pseudosurface waves and the null angles.

During our search for nulls in various anisotropic materials, we were unable to find a null in the hexagonal materials under consideration. But, all the tetragonal materials we examined, exhibited a null. As indicated by Farnell ${ }^{20}$ the ratio $\beta=2 c_{66} /\left(c_{11}-c_{12}\right)$ plays a similar role with the tetragonal

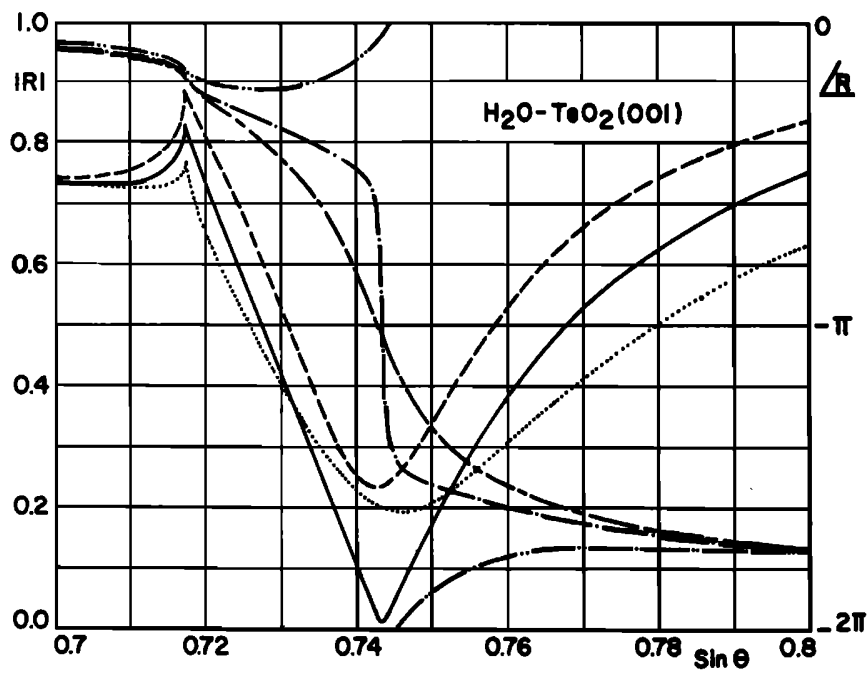

FIG. 14. Reflection coefficient magnitude at a $\mathrm{H}_{2} \mathrm{O}-\mathrm{TeO}_{2}(001)$ interface near the null as a function of incidence angle for azimuthal angles of $25.65^{\circ}$ (dotted), $28.65^{\circ}$ (solid), $31.65^{\circ}$ (dashed) and phase for $25.65^{\circ}$ (dash-dotdot), $28.65^{\circ}$ (dash-dot), $31.65^{\circ}$ (dash-long dash).

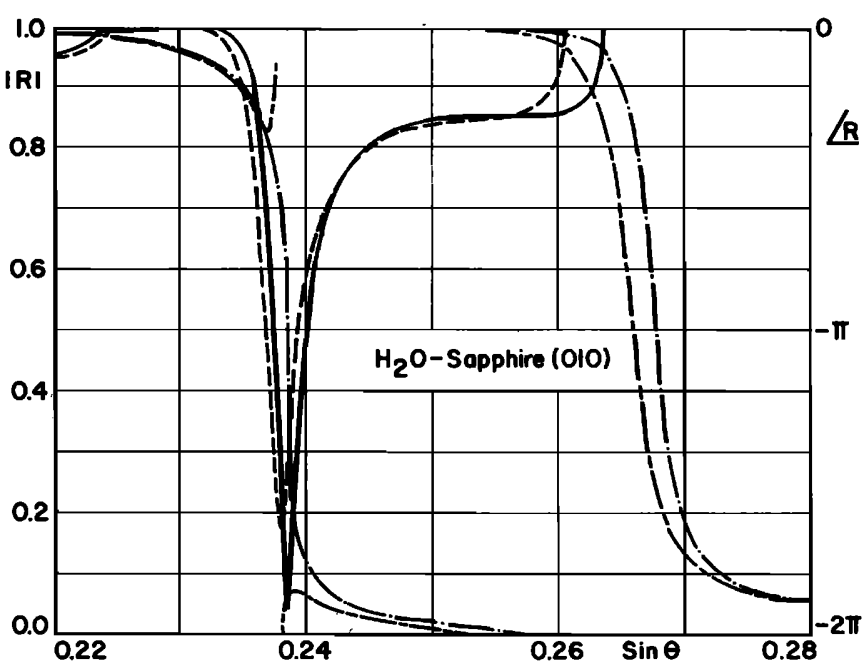

FIG. 16. Reflection coefficient magnitude at $\mathrm{a}_{2} \mathrm{O}$-sapphire (010) interface near the null as a function of incidence angle for azimuthal angles of $3.19^{\circ}$ (solid), $9.0^{\circ}$ (dashed), and phase for $3.19^{\circ}$ (dash-dot), $9.0^{\circ}$ (dashlong dash). 
crystals as did $\eta$ for the cubic crystals. Out of the three planes of the quartz crystal, only the $Y-Z(100)$ plane gave a null in the reflection coefficient. This is consistent with previous experimental results ${ }^{14,15}$ that showed that only the (100) plane of quartz has a pseudosurface wave. Moreover, the reported phase velocity and the azimuthal angle agree with our findings. As Table IV indicates only the $X-Z(010)$ planes of sapphire and $\mathrm{LiNbO}_{3}$ exhibit nulls. Note that, in all computations piezoelectricity is neglected. So, our results apply only approximately for piezoelectric materials.

\section{CONCLUSION}

In this article, the results of numerical calculations on the acoustical reflection problem at the liquid-anisotropicsolid interface are presented and reflection coefficient plots for various solids are obtained. The null found in the reflection coefficient for some materials corresponds to an efficient excitation of a pseudosurface wave in the solid material. Among the anisotropic materials we examined those surfaces that support pseudosurface waves seem to have a null in the reflection coefficient. It is possible to some extent to classify the crystalline materials for the existence of the pseudosurface waves. In general, materials which are nearly isotropic do not support pseudosurface waves, and hence, they do not have nulls in the reflection coefficient. In particular, we have determined that the presence and angular extent of the null angle depend on the anisotropy factor in cubic materials. For (001) and (111) planes a high anisotropy factor will correspond to a wide null in the reflection coefficient. On the contrary, the (011) plane of a cubic material requires an anisotropy factor smaller than unity for the presence of the null angle. We were unable to find nulls in a few materials we examined that belong to the hexagonal crystal class. All tetragonal materials under investigation showed nulls at their (001) planes.

The presence of the null angle provides a wideband matching from a bulk wave in the liquid to a shear wave in the solid. By reciprocity the same should hold true when a QS wave is incident on the solid-liquid interface from the solid side at the proper angle. This fact can be used as a method for coupling acoustic power from one side to the other without any bandwidth limitation. One disadvantage of this method is that this coupling occurs only for a very narrow angular aperture, hence, one has to use wide beams with a narrow angular spectrum.

\section{ACKNOWLEDGMENT}

Some of this work was done while the first two authors were with the Electrical and Electronics Engineering De- partment of Middle East Technical University in Ankara, Turkey.

'L. M. Brekhovskikh, Waves in Layered Media (Academic, New York, 1980).

${ }^{2} \mathrm{G}$. Mott, "Reflection and refraction coefficients at a fluid-solid interface," J. Acoust. Soc. Am. 50, 819-829 (1971).

${ }^{3}$ W. G. Neubauer, "Ultrasonic reflection of a bounded beam at Rayleigh and critical angles for a plane liquid-solid interface," J. Appl. Phys. 44, 48-55 (1973).

${ }^{4}$ E. G. Henneke, II "Reflection-refraction of a stress wave at a plane boundary between anisotropic media," J. Acoust. Soc. Am. 51, 210-217 (1972).

${ }^{5}$ E. G. Henneke, II and G. L. Jones, "Critical angle for reflection at a liquid-solid interface in single crystals," J. Acoust. Soc. Am. 59, 204-205 (1976).

'B. A. Auld, Acoustic Fields and Waves in Solids (Wiley-Interscience, New York, 1973), Vols. 1 and 2.

${ }^{7}$ G. L. Jones and E. G. Henneke, II, "Reflection of stress waves at a free boundary in quartz single crystals," IEEE Trans. Sonics Ultrason. SU-20, 267-274 (1973)

${ }^{8} \mathrm{~A}$. Atalar, "Reflection of ultrasonic waves at a liquid-cubic-solid interface," J. Acoust. Soc. Am. 73, 435-440 (1983).

${ }^{9}$ M. G. Somekh, G. A. D. Briggs, and C. Ilett, "The effect of elastic anisotropy on contrast in the scanning acoustic microscope," Philos. Mag. A 49, 179-204 (1984).

${ }^{10} \mathrm{G}$. M. Crean and A. Waintal, "Average Rayleigh-wave velocity of a computer-simulated crystallographic plane,” J. Appl. Cryst. 19, 181-187 (1986).

"V. R. Velasco and F. Garcia-Moliner, "Theory of surface waves in anisotropic cubic crystals," J. Phys. C: Solid State Phys. 13, 2237-2256 (1980).

${ }^{12}$ S. I. Rokhlin, T. K. Bolland, and L. Adler, "Reflection and refraction of elastic waves on a plane interface between two generally anisotropic media," J. Acoust. Soc. Am. 79, 906-918 (1986).

${ }^{13}$ S. I. Rokhlin, T. K. Bolland, and L. Adler, "Splitting of domain of angles for incident wave vectors in elastic anisotropic media," J. Appl. Phys. 59, 3672-3677 (1986).

${ }^{14} \mathrm{H}$. Engan, K. A. Ingebrigtsen, and A. Tonning, "Elastic surface waves in $\alpha$-quartz: Observation of leaky surface waves," Appl. Phys. Lett. 10, 311313 (1967).

${ }^{15}$ L. V. Verevkina, L. G. Merkulov, and D. A. Tursunov, "Surface waves in a quartz crystal," Sov. Phys. Acoust. 12, 254 (1967).

${ }^{16} \mathrm{~F}$. R. Rollins, "Ultrasonic examination of liquid-solid boundaries using a right-angle reflector technique," J. Acoust. Soc. Am. 44, 431-434 (1968).

${ }^{17}$ F. R. Rollins, T. C. Lim, and G. W. Farnell, "Ultrasonic reflectivity and surface wave phenomena on surfaces of copper single crystals," Appl. Phys. Lett. 12, 236-238 (1968).

${ }^{18}$ O. I. Diachok, R. J. Hallermeier, and W. G. Mayer, "Measurements of ultrasonic surface wave velocity and absorptivity on single-crystal copper," Appl. Phys. Lett. 17, 288-299 (1970).

${ }^{19}$ T. C. Lim and G. W. Farnell, "Character of pseudo surface waves on anisotropic crystals," J. Acoust. Soc. Am. 45, 845-851 (1969).

${ }^{20} \mathrm{G}$. W. Farnell, "Properties of elastic surface waves," in Physical Acoustics, edited by W. P. Mason and R. N. Thurston (Academic, New York, 1970), Vol. 6, pp. 109-166.

${ }^{21} \mathrm{~A}$. G. Every, "Pseudosurface wave structures in phonon imaging," Phys. Rev. B 33, 2719-2732 (1986). 